\title{
Reward-related brain function as a predictor of treatment response in adolescents with major depressive disorder
}

\author{
Erika E. Forbes, Thomas M. Olino, Neal D. Ryan, Boris Birmaher, \\ David Axelson, Donna L. Moyles, and Ronald E. Dahl \\ University of Pittsburgh, Pittsburgh, Pennsylvania
}

\begin{abstract}
The present study provides preliminary evidence that pretreatment reward-related brain function in the striatum and medial prefrontal cortex (PFC) could have relevance for predicting both final level and rate of change of clinical characteristics in adolescents with major depressive disorder. Adolescents with depression underwent a functional MRI scan during a monetary reward task, participated in an 8-week open trial of cognitive behavioral therapy (CBT) or CBT plus selective serotonin reuptake inhibitor, and completed reports of anxiety and depressive symptoms before, during, and after treatment. Clinicians rated adolescents' improvement and severity at the same time points. Growth models were used to examine change in clinical characteristics and its association with brain function. Severity, anxiety symptoms, and depressive symptoms decreased over treatment. Final levels of severity and anxiety symptoms were associated with pretreatment striatal reactivity, and rate of anxiety symptom reduction was associated with greater striatal reactivity and lower medial PFC reactivity.
\end{abstract}

Research on the etiology, pathophysiology, and clinical course of depression can contribute to the development of new treatments and the refinement of existing treatments. Importantly, because variability exists both in response to empirically supported treatments for depression and in affect-related brain function among people with depression, there is the potential to determine whether individual differences in affect-related brain function are associated with response to treatment. Thus, research on the neural substrates of depression can possibly reduce the heterogeneity of treatment response and contribute to the goal of improving outcomes by personalizing treatment.

Because depression is considered to be a disorder of affect regulation (Forbes \& Dahl, 2005; Gross \& Muñoz, 1995), neural response to affective stimuli could indicate alterations in many processes relevant to treatment response, including subjective experience of affect-eliciting events, ability to engage interpersonally in psychosocial treatment, and functioning of neural circuits potentially influenced by psychosocial or pharmacologic treatments. Studies have begun to address affect-related brain function as a predictor of treatment response, with stimuli including sad faces (Costafreda, Khanna, Mourao-Miranda, \& Fu, 2009; Fu et al., 2008), fearful faces (Salvadore et al., 2009), happy faces (Fu et al., 2007), positive and negative affective words (Siegle, Carter, \& Thase, 2006), and positive and negative affective faces (Sheline et al., 2001; Whalen et al., 2008). These studies have addressed improvement with cognitive behavioral therapy (CBT; e.g., Fu et al.,
2008) and pharmacotherapy (e.g., Salvadore et al., 2009), focusing on both depression (e.g., Siegle et al., 2006) and anxiety disorders (e.g., Whalen et al., 2008).

Adolescence is a particularly important point in the life span for understanding the implications of brain function for treatment response, since intervention early in the clinical course of depression - and during brain development - could be especially helpful in preventing future episodes. Adolescence is a time of great increase in the incidence of depression (Angold, 1988; Lewinsohn, Clarke, Seeley, \& Rohde, 1994), and many adults with depression experienced their first episode during adolescence (Lewinsohn, Rohde, Klein, \& Seeley, 1999; Weissman et al., 1999).

Reward-related brain function could be a particularly fruitful target for investigating affect-related brain function as a predictor of treatment response in adolescent depression. Reduced reward-related striatal function has been observed in adolescents and adults with depression (Epstein et al., 2006; Forbes, Hariri, et al., 2009; Keedwell, Andrew, Williams, Brammer, \& Phillips, 2005; Surguladze et al., 2005), and it is postulated to be associated with affective and motivational features of the disorder (Davey, Yücel, \& Allen, 2008; Forbes \& Dahl, 2005). The association of reward-related brain function with treatment response remains to be examined, since the most relevant study of affect-related brain function as a predictor of treatment response in adolescents focused on threat-related brain function and anxiety disorders (McClure et al., 2007). 
To date, three lines of evidence suggest that functioning in reward-related brain areas could be important in treatment response in adolescents with depression. First, research on psychosocial treatment suggests that improvement of positive affect - a larger construct that includes reward processing - is part of the response to treatment in adult depression. Specifically, CBT is associated with increased level of positive affect in adults whose depression improves significantly (Kring, Persons, \& Thomas, 2007). Second, in adults with depression, amphetamine challenge, which exerts its effects through dopamine release in the ventral striatum, elicits greater subjective sensitivity to the rewarding effects of amphetamine and unusual striatal functioning (Tremblay et al., 2005). This finding suggests that striatal functioning is reduced in depression and reflects the emerging consensus that functioning of the dopamine system - which is critical to reward processing - contributes to the pathophysiology of depression (Dunlop \& Nemeroff, 2007). Third, stimulation of reward-related areas in the brain can elicit improvement in adults with recurrent depression. Deep brain stimulation (DBS) to the ventral striatum has been demonstrated as an efficacious treatment in adults with severe, recurrent depression (Schlaepfer et al., 2008).

One possible explanation for these results is that individual differences in reward-related striatal function at baseline can indicate which depressed patients have the flexibility in the function of reward-related neural systems to respond to treatment. This hypothesis is consistent with a phenomenological finding in adolescent depression: A relatively higher level of subjective positive mood in natural environments in adolescents who are currently in a depressive episode is associated with lower likelihood of recurrence (Silk et al., 2010). This finding suggests that higher positive affect can be protective (e.g., by facilitating hope despite depression) or, possibly, promote improvement.

Although often neglected, comorbidity with anxiety is worthy of attention in research on the neural predictors of treatment response in depression. Anxiety disorders are common in young people with depression, and the two types of disorders have shared familial risk and common genetic liability (Williamson, Forbes, Dahl, \& Ryan, 2005). Anxiety and depression appear to have distinctive features, but there is considerable overlap between them (Olino, Klein, Lewinsohn, Rohde, \& Seeley, 2008). Little conceptual attention has been paid to associations between anxiety and reward processing, but recent evidence suggests that, contrary to the pattern of findings in depression, anxiety traits and disorders in adolescents involve enhanced reward-related functioning in the striatum (Bar-Haim et al., 2009; Guyer et al., 2006) and orbitofrontal cortex (Forbes et al., 2006). For adolescents with comorbid depression and anxiety, it is difficult to predict whether reward would elicit more or less reactivity in reward-relevant areas such as the striatum, since depression is associated with decreased reactivity and anxiety with increased reactivity. One possibility is that adolescents with depression and comorbid anxiety do not exhibit the decreased reward-related reactivity that adolescents with "pure" depression exhibit.

In addition, a key methodological issue in examining neural predictors of treatment response is the operationalization of treatment response. Typically, treatment response has been measured by change in symptom level from pre- to posttreatment (e.g., Siegle et al., 2006) or by ratings of improvement posttreatment (e.g., McClure et al., 2007), rather than by change in severity or symptoms before, during, and after treatment. Psychopathology researchers emphasize that treatment response is best measured across multiple time points (Willett, 1997), however. As a result, methods such as growth curve modeling, which allow the examination of improvement over time, have become more widely used in treatment outcome research (Lutz, Martinovich, \& Howard, 1999). It is also valuable to take a multimeasure approach that includes several aspects of clinical state and assesses response from several perspectives. Clinical factors worth considering are both those that are global (i.e., related to general functioning or behavior across several domains) and those that are specific (i.e., relevant to the symptoms and signs of the disorder). Perspectives worth considering are those of the clinicians assessing or treating participants and, of course, those of the participants themselves (Spielmans \& McFall, 2006).

In the present study, we examined reward-related brain function in relation to change in self-reported depressive and anxiety symptoms and clinician-reported clinical features in adolescents with major depressive disorder, most of whom had comorbid generalized anxiety disorder. On the basis of the growing literature on altered reward-related striatal function in depression, we predicted that greater striatal reactivity at baseline would predict both lower levels of symptoms and less severity at the end of treatment (intercept) and faster decrease in symptoms and severity during treatment (slope). This prediction was somewhat tentative, however, because of the presence of comorbid anxiety disorders in the sample and reports of decreased striatal reactivity in the small literature on reward functioning in anxiety. Because both depression and anxiety have been associated with altered striatal functioning, and because we employed a functional MRI task that reliably elicits striatal reactivity (Delgado, Nystrom, Fissell, Noll, \& Fiez, 2000; Forbes, Hariri, et al., 2009), we focused on this region as relevant to our research question.

In addition, we examined reward-related reactivity of the medial prefrontal cortex (mPFC) as a predictor of treatment response. Although we did not have strong hypotheses about the association of $\mathrm{mPFC}$ reactivity and treatment response, several findings led us to investigate whether low reactivity in this region would predict better response to treatment. First, we have reported previously that adolescents with depression have greater $\mathrm{mPFC}$ reactivity to reward than do comparison adolescents (Forbes, Hariri, et al., 2009). Second, findings on affect-related brain function as a predictor of treatment response in depression have indicated that higher medial prefrontal function to sad stimuli before treatment is related to nonremission with 
CBT (Costafreda et al., 2009). Third, research on default mode network functioning indicates that adult depression is associated with less disengagement of the default mode network during affective processing (Sheline et al., 2009). The medial PFC is included in the default mode networka set of brain regions in which activity is high during a resting or self-referential state but decreases during cognitive engagement (Raichle \& Snyder, 2007) - and has been implicated in depression-related alterations in the functioning of this network. Given this support for higher mPFC reactivity in depression and in poor response to treatment, we predicted that greater $\mathrm{MPFC}$ reactivity before treatment would be associated with higher levels of severity and symptoms after treatment (intercept) and slower decrease in symptoms and severity across treatment (slope).

\section{METHOD}

\section{Participants}

The participants were 13 adolescents $10-16$ years of age $(M=$ $12.9 ; S D=2.3 ; 69.2 \%$ female; $92.3 \%$ European American), all of whom had major depressive disorder (MDD). Ten MDD participants had comorbid generalized anxiety disorder (GAD). Of those 10 with $\mathrm{MDD}$ and GAD, 3 also had diagnoses of separation anxiety disorder, 1 also had social phobia, 1 also had panic disorder, and 1 had both social phobia and panic disorder. The participants were free of psychotropic medications, nicotine, and illicit drugs. Participants were recruited from outpatient clinics at Western Psychiatric Institute and Clinic, Pittsburgh, and through advertisements. MDD diagnoses were confirmed by a face-to-face interview with a child psychiatrist. After a complete description of the study, written informed consent was obtained from all the parents and from the participants 14 years of age and older; younger participants provided verbal assent.

\section{Measures}

Diagnosis. Diagnoses were determined through use of the Schedule for Affective Disorders and Schizophrenia for SchoolAge Children-Present and Lifetime Version (K-SADS-PL; Kaufman et al., 1997). This structured clinical interview is reliable and valid and has been used widely in studies of adolescent affective disorders.

Symptoms, severity, and improvement. Symptom, severity, and improvement measures were selected on the basis of their comparability with other studies of brain function predicting treatment response in adolescent affective disorders (e.g., McClure et al., 2007), wide use in adolescent depression research, and sound psychometric properties. The participants, parents, and clinicians completed measures of symptoms, severity, and improvement during the initial diagnostic assessment and then after four treatment sessions (see below).

The participants and their parents completed the Screen for Childhood Anxiety and Related Disorders (Birmaher et al., 1999) and the Mood and Feelings Questionnaire (MFQ; Angold, Erkanli, Silberg, Eaves, \& Costello, 2002) to report symptoms of anxiety and depression, respectively. Both are widely used measures with excellent psychometric properties. The MFQ, for example, has been evaluated carefully for its psychometric features (Katon, Russo, Richardson, McCauley, \& Lozano, 2008; Wood, Kroll, Moore, \& Harrington, 1995). Clinicians who provided treatment completed the Clinical Global Impressions Severity (CGI-S; all five time points) and Improvement (CGI-I; last four time points) scales (Guy, 1976). The CGI-S is a single-item 7-point scale, with higher scores reflecting greater severity. The CGI-I is a single-item 8-point scale, with lower scores reflecting greater improvement. The CGI scales have been used in large and rigorous randomized controlled trials of psychosocial and pharmacologic treatments for adolescent depression (e.g.,
March et al., 2004). Clinicians administering the CGI-S and CGI-I scales compared and discussed their ratings during training, but formal reliability was not computed for ratings.

Reward processing. The fMRI task was an adaptation of a card-guessing paradigm developed to probe striatal response to feedback associated with monetary reward (Delgado et al., 2000). In our event-related task, each trial included both an anticipation period and an outcome period, and the participants received win, loss, or no-change feedback for each trial. The participants were told that their performance would determine a monetary reward to be received after the scan.

Trials were presented in pseudorandom order with predetermined outcomes. During each 27-sec trial, participants had $3 \mathrm{sec}$ to guess, via buttonpress, whether the value of a visually presented card with a possible value of 1-9 was higher or lower than 5 (middle or index finger, respectively). After a choice was made, the trial type (reward or loss) was presented visually for $12 \mathrm{sec}$ (anticipation). This was followed by the "actual" numerical value of the card $(500 \mathrm{msec})$, outcome feedback (a green upward-facing arrow for win, a red downward-facing arrow for loss, or a yellow circle for neutral feedback; $500 \mathrm{msec}$ ), and a crosshair presented for $11 \mathrm{sec}$ (outcome). There were no trials involving anticipation of a neutral outcome. That is, neutral feedback trials were those with no-win or no-loss outcomes (i.e., no monetary increase or decrease). Trials were presented in four runs, with 12 trials per run and a balanced number of trial types within runs (i.e., 6 possible-win and 6 possible-loss trials, with a total of three win, three loss, and three neutral outcomes; see Forbes, Hariri, et al., 2009, for additional details).

The participants were told that they would receive $\$ 1$ for each win, lose 50 cents for each loss, and experience no earnings change for neutral outcomes. The participants were unaware of the fixed outcome probabilities and were led to believe that performance would determine net monetary gain. The participants' engagement and motivation to perform well were maintained by verbal encouragement during practice and between runs. The participants' mean reaction time during reward trials was $8,560 \mathrm{msec}(S D=23,098.50)$. Reaction time was uncorrelated with age, presence of comorbid anxiety disorder, medication status, and severity variables.

\section{Procedure}

The local institutional review board approved the study. The participants' assessment included diagnosis, symptom questionnaires, and assignment to treatment type. The MRI scan was conducted at the time of the diagnostic assessment, which was the week before treatment began. Before the scan, the participants practiced the paradigm and experienced the scanning environment through a simulator to ensure familiarity with the environment and to gauge comfort. The participants received $\$ 60$ for the scan, plus $\$ 15$ for "playing the game."

Diagnosis. Diagnoses were determined through administration of the K-SADS-PL (Kaufman et al., 1997). Each participant and a parent (or guardian) were interviewed separately by a bachelor'slevel research specialist trained according to local diagnostic reliability standards. Reliability for depressive and anxiety diagnoses was $>90 \%$ and was maintained through monthly department-wide diagnostic reviews. A child psychiatrist provided best-estimate diagnoses. All the participants were in a current episode of MDD.

Treatment. Treatment involved an 8-week open treatment protocol with CBT $(n=7)$ or CBT combined with pharmacotherapy using a selective serotonin reuptake inhibitor $(n=6)$. Participants were not assigned to treatment conditions, but they were instead offered three options: CBT only, CBT plus pharmacotherapy, or pharmacotherapy only. None of the participants chose pharmacotherapy only. Choice of treatment was not related to age, gender, race, symptom level, or clinician-rated general clinical severity (all $F_{\mathrm{s}}<1$, all $\left.\chi^{2} \mathrm{~s}<2.0, p \mathrm{~s}>.20\right)$. CBT was provided by six therapists.

CBT was provided through the Services for Teens at Risk (STAR) Clinic at Western Psychiatric Institute and Clinic, and it was tailored to each participant and guided by techniques developed by 
Brent et al. (1997). CBT was provided by trained PhD psychologists and masters-level social workers or nurses. Pharmacotherapy was provided by child psychiatrists assisted by psychiatric research nurses.

For pharmacotherapy, the participants were scheduled to have face-to-face weekly meetings with the treating psychiatrist and a psychiatric research nurse for the 1st month of the study. During appointments, psychiatrists observed clinical worsening or improvement and possible side effects. On the $1 \mathrm{st}$, $3 \mathrm{rd}, 5$ th, and 7 th weeks of treatment, meetings lasted approximately $15 \mathrm{~min}$. On the $2 \mathrm{nd}$, 4 th, 6th, and 8th weeks of treatment, the participants had a 60-min appointment that also included standard self-reports and clinician rating scales. Medications used in pharmacotherapy were citalopram (10-40 mg) or fluoxetine (5-25 mg). Starting doses were the smallest that were likely to be tolerated by all the participants and would be considered the minimum therapeutic dose. For fluoxetine, $5 \mathrm{mg}$ was selected as the starting dose for participants weighing $<30 \mathrm{~kg}$, and $10 \mathrm{mg}$ was selected for participants weighing $\geq 30 \mathrm{~kg}$. If the participants did not show significant clinical improvement after 2 weeks and were tolerating the dose, the dose was then increased to what was likely to be therapeutic for the majority of the participants (e.g., for fluoxetine, $10 \mathrm{mg}$ for participants weighing $<30 \mathrm{~kg}$, and $20 \mathrm{mg}$ for participants weighing $\geq 30 \mathrm{~kg}$ ). After 4 weeks, if the participant was not responding to this dose, the treating psychiatrist increased the dose as tolerated. In addition, if a participant had an initial brief, but nonsustained response to fluoxetine (e.g., was much improved at week 2 but regressed back to baseline on subsequent visits), the psychiatrist increased the fluoxetine dose as tolerated. The dose of medication could be reduced at any time for side effects at the discretion of the treating psychiatrist.

A total of 6 participants did not complete treatment. Dropout tended to occur during the second half of treatment, with 10 participants completing severity measures at Time 3 . We did not further assess participants or parents who decided not to continue for the entire course of treatment. All completers had comorbid anxiety disorders, and 4 were in the combined CBT and medication group. Completers and noncompleters did not differ significantly on gender, age, treatment modality, presence of comorbid anxiety disorders, or symptom severity at baseline. Clinician-rated improvement was not assessed at baseline, but completers and noncompleters did not differ in improvement at Week 3. Completers had higher pretreatment depressive symptoms than did noncompleters $(M=33.20, S D=6.94$, and $M=21.00, S D=8.76$, respectively; $t=2.58, p<.05)$.

Clinical change over time. Symptoms, severity, and improvement were measured at five time points: before treatment (hereafter, Time 1) and at Treatment Weeks 2, 4, 6, and 8 (Times 2-5). Because of declining to complete questionnaires, time constraints, or failure to complete the 8 weeks of treatment, depressive symptom data were missing for 1 participant at Time 1, 1 at Time 2, 5 at Time 3, 6 at Time 4 , and 8 at Time 5 . Anxiety symptom data were missing for 1 participant at Time 1,3 at Time 2, 5 at Time 3, 6 at Time 4, and 8 at Time 5. Clinician severity ratings were missing for 2 participants at Time 1, 2 at Time 2, 3 at Time 3, 5 at Time 4, and 7 at Time 5. Similarly, clinician improvement ratings were missing for 1 participant at Time 2, 3 at Time 3, 5 at Time 4, and 7 at Time 5. Analyses included all the available data for each time point.

\section{BOLD fMRI Acquisition, Processing, and Analysis}

Each participant was scanned using a Siemens 3T Allegra scanner. BOLD functional images were acquired with a gradient echo EPI sequence and covered 34 axial slices ( $3 \mathrm{~mm}$ thick) beginning at the cerebral vertex and encompassing the entire cerebrum and the majority of the cerebellum $(\mathrm{TR} / \mathrm{TE}=2,000 / 25 \mathrm{msec}, \mathrm{FOV}=$ $20 \mathrm{~cm}$, matrix $=64 \times 64)$. Scanning parameters were selected to optimize BOLD signal quality while maintaining a sufficient number of slices to acquire whole-brain data. Before the collection of fMRI data for each participant, a reference EPI scan was acquired and visually inspected for artifacts (e.g., ghosting) and good signal across the entire volume. The data from all the participants were clear of such problems.

Whole-brain image analysis was conducted using SPM5 (www .fil.ion.ucl.ac.uk/spm). For each scan, images for each participant were realigned to the first volume in the time series to correct for head motion. The motion correction criterion was set at $<4 \mathrm{~mm}$, as is our practice with young people from clinical populations (Forbes, Hariri, et al., 2009). Realigned images were spatially normalized into Montreal Neurological Institute stereotactic space using a 12-parameter affine model, then smoothed to minimize noise and residual difference in gyral anatomy with a Gaussian filter set at $6 \mathrm{~mm}$ full-width at half-maximum. Voxel-wise signal intensities were ratio-normalized to the whole-brain global mean.

Preprocessed data were analyzed using second-level random effect models that account for both scan-to-scan and participant-toparticipant variability to determine task-specific regional responses. Analyses focused on data for the first run, since these data were less likely to reflect the influence of fatigue, habituation, boredom, and frustration with task length. Habituation was a particular concern, because striatal response to reward tends to diminish with repeated experience of a reward (e.g., Koob \& Le Moal, 2008). Although focusing on the first run, rather than on the entire task, limited the number of trials included in the analyses, it reduced habituation effects and also increased the number of participants with movementfree data available for analyses. Consistent with concerns about change in response over the task, we found considerable variability in reward response when comparing response during the first run to response during the entire task: Stability for striatal reactivity was .60 for reward anticipation and .60 for reward outcome; stability for $\mathrm{mPFC}$ reactivity was -.50 for anticipation and .13 for outcome.

Because a priori hypotheses concerned the role of reward processing in depression, analyses focused on reward, rather than on loss conditions. For each participant and scan, predetermined condition effects at each voxel were calculated using a $t$ statistic, producing a statistical image for two contrasts: reward anticipation $>$ baseline and reward outcome $>$ baseline. Individual contrast images were then used in second-level analyses to determine mean anticipationrelated and outcome-related reward response, using one-sample $t$ tests (see Figure 1). All tests were set to a threshold of $p<.05$, required to have a minimum extent of 10 contiguous voxels, and corrected for multiple comparisons using false discovery rate across activation clusters of interest based on region of interest (ROI) analyses. Two ROIs were included: (1) a striatal ROI, as in Forbes, Brown, et al. (2009), and (2) an mPFC ROI. The striatal ROI was a sphere with 20 -mm radius, centered on Talairach coordinates $x=0, y=10$, $z=-10$ and encompassing the entire bilateral ventral striatum and adjacent regions of the caudate. The mPFC ROI was defined as a sphere with 25-mm radius, centered on Talairach coordinates $x=0$, $y=44$, and $z=18$ and encompassing BA32 and the medial areas of BA9 and BA10. Both ROIs were constructed using the WFU PickAtlas Tool (v2.4). Results of one-sample $t$ tests were then extracted for inclusion in growth models estimating level and change of symptoms, severity, and improvement.

\section{Growth Models}

We estimated symptom and impairment reduction within a linear growth modeling framework. In contrast to pre-post designs, in which indicators of change are measured only before and after treatment, growth models had several advantages for analyzing the data in the present study. Growth models are well suited to analyzing data sets that include more than two assessments of symptom scores, and unlike pre-post analyses, they accommodate missing data by including all available scores. In the current study, growth curve models explicitly included time as part of the model, producing an average rate of change over the course of treatment; were used to estimate missing data; and allowed for examinations of predictors of both symptom severity and rate of symptom change in the same model. Although the present study's sample size was relatively small 
A
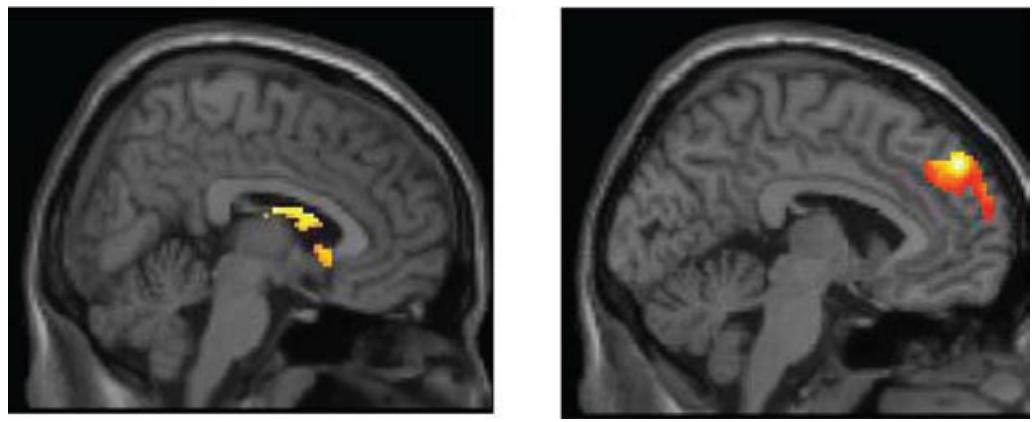

B
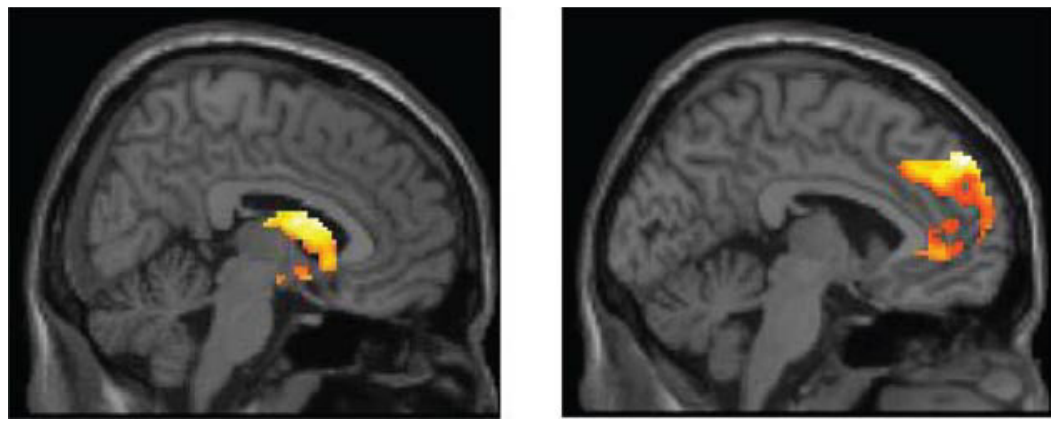

Figure 1. Main effects of reward processing task on reactivity in clusters in the striatum (on the left) and medial prefrontal cortex (on the right) in adolescents with major depressive disorder during reward anticipation (A) and reward outcome (B).

for growth curve modeling, its inclusion of up to five observations per case made the total number of data points adequate for the application of growth modeling (Maas \& Hox, 2005). ${ }^{1}$

Initial models define score change on the basis of two primary parameters, intercept and slope, both of which have mean and variance estimates. The intercept refers to an identified location of the growth pattern - here, at the end point of treatment. The intercept reflected each individual end point of treatment, since not all the participants completed the full 8 weeks. The mean value of the intercept is an estimated value of the overall sample based on all assessment points in the model, adjusted for the slope. The intercept parameter is further characterized by a variance estimate that identifies whether there is significant variation in the outcome variable at the specified time value. The mean value of the slope is the average rate of change over time in the specified outcome. The slope is also characterized by a variance parameter that identifies whether there is interindividual variation in the rate of change in levels of the outcome variable. Importantly, predictors of intercept and slope may be examined only if the parameter has significant variance (Raudenbush \& Bryk, 2002). Linear growth models were estimated using Mplus 5.2, specifying the two-level and random analysis options (Muthén \& Muthén, 2006). We examined treatment as a function of real time (in weeks), as opposed to number of therapeutic contacts. However, the results were largely the same for each conceptualization of time.

The main data analyses were conducted in three stages. First, within-group $t$ tests were conducted on pretreatment fMRI data in SPM5 to identify voxels of maximum activation during reward anticipation and reward outcome in two primary ROIs: the striatum and mPFC. Second, mixed-effects growth curve models were fitted to the data that examined the level-one associations between time and symptom scores (i.e., change in symptoms or improvement with time). Because symptom scores were nested within person, the relationship between time (measured in weeks) and symptom scores was a random effect. Third, linear mixed model analyses were conducted using Mplus 5.2 (Muthén \& Muthén, 2006) with fMRI predictors as fixed effects in relation to parameters from the growth curve models (e.g., striatal reactivity during reward anticipation predicting final level of depressive symptoms or change in depressive symptoms over time). Although a log transformation is sometimes applied to clinical data to account for varying rate of improvement during treatment (e.g., faster improvement in early weeks of treatment than in later weeks), this approach did not improve our ability to detect associations and did not seem empirically appropriate, because the rate of change appeared to be fairly consistent across measures (see Figure 2). Since the analyses are preliminary, we interpret trends, as well as reporting significant findings.

\section{RESULTS}

\section{Preliminary Analyses}

Preliminary analyses examined (1) the association between age and reward-related brain function; (2) possible differences in treatment response based on medication status, comorbid anxiety disorder, and age; and (3) correlations between brain function variables. Age was uncorrelated with striatal or $\mathrm{mPFC}$ reactivity during either reward anticipation or reward outcome. Although sample size prevented the inclusion of medication status as an additional predictor in the growth curve models reported below, a separate model was conducted to ex- 
A

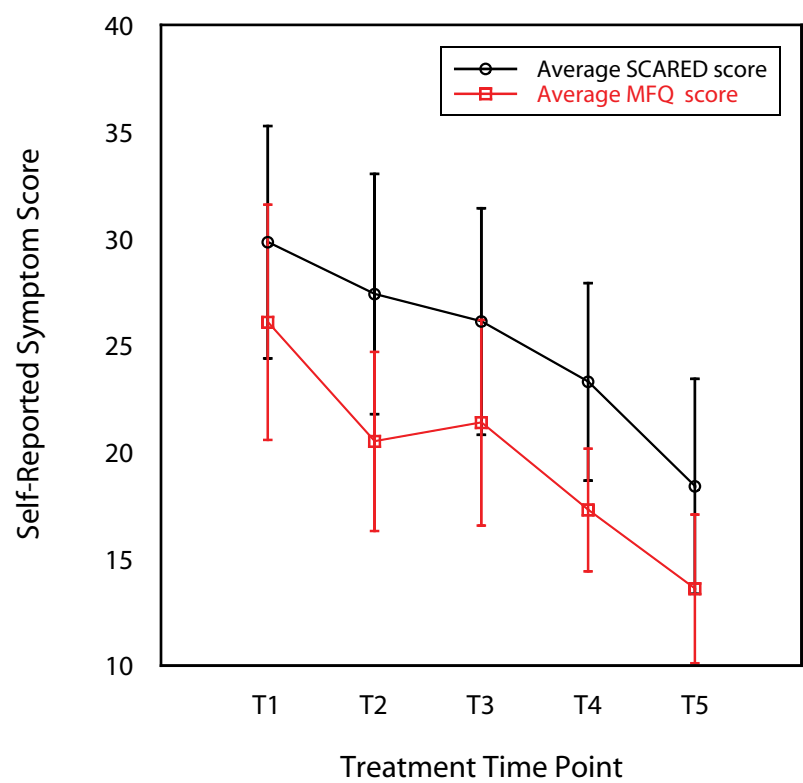

B

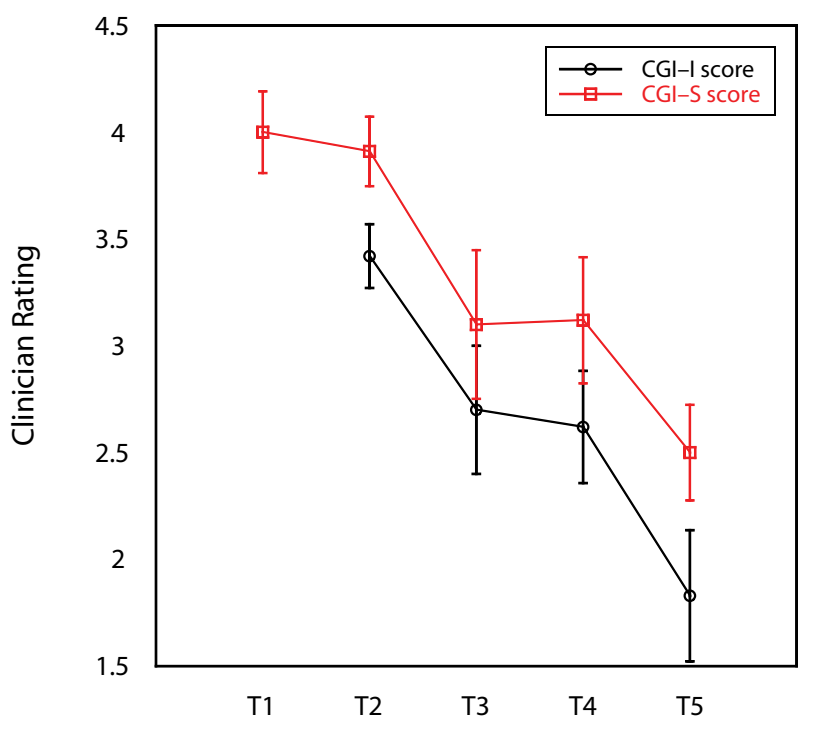

Treatment Time Point

Figure 2. Mean level of self-reported anxiety and depressive symptoms (A) and clinician-rated severity and improvement (B) across five time points in an 8-week treatment course for adolescents with major depressive disorder. Error bars represent 1 standard error of the mean at each time point. Improvement is not assessed at pretreatment, so it is reported only for four time points. MFQ, Mood and Feelings Questionnaire (depressive symptom measure); SCARED, Screen for Childhood Anxiety and Related Disorders (anxiety symptom measure); CGI-S, Clinical Global Impression-Severity; CGI-I, Clinical Global ImpressionImprovement; T1, pretreatment; T2, Treatment Session 2; T3, Treatment Session 4; T4, Treatment Session 6; T5, Treatment Session 8 (posttreatment). amine medication status (CBT only or CBT plus pharmacotherapy) as a predictor of symptom change. The combination of CBT and medication (vs. CBT alone) was associated with lower depressive $(B=-10.89$, $S E=4.79, p<.05)$ and anxiety $(B=-13.96, S E=$ $5.09, p<.01)$ symptoms at discharge. Medication status was not associated with general clinical severity level at discharge or rate of change in anxiety symptoms. The participants with comorbid anxiety disorders had higher general clinical severity $(B=42, S E=0.21, p<.05)$ and depressive symptoms $(B=10.68, S E=3.49, p<$ $.01)$ at discharge. In addition, comorbid anxiety disorder was associated with a faster rate of reduction in anxiety symptoms $(B=-2.74, S E=0.77, p<.001)$. Despite these findings for treatment modality and comorbid anxiety, it was not possible to conduct the main analyses separately by either variable because of the limited sample size. Age was not associated with any measure of treatment response. Within each brain ROI, correlations across task condition were strong: Striatal reactivity during reward anticipation was moderately correlated with striatal reactivity during reward outcome $(r=.55, p=$ $.05)$, and $\mathrm{mPFC}$ reactivity during anticipation was highly correlated with $\mathrm{mPFC}$ reactivity during reward outcome $(r=.75, p<.01)$. Within each task condition, striatal reactivity and $\mathrm{mPFC}$ reactivity were modestly correlated for reward anticipation $(r=-.27, p<.40)$ and strongly correlated for reward outcome $(r=.75, p<.005)$.

\section{Main Effect of Task in the Striatum and mPFC}

As is shown in Figure 1, striatum ROI analyses for the main effects of task indicated activation in the right caudate for both reward anticipation $\left(t=4.49, p_{\mathrm{FDR}}<.05\right.$; 682 voxels; Talairach coordinates: $6,5,16)$ and reward outcome $\left(t=4.32, p_{\mathrm{FDR}}<.05 ; 881\right.$ voxels; Talairach coordinates: $6,12,12)$. ROI analyses for the mPFC indicated activation in medial Brodmann Area (BA) 9 for both reward anticipation $\left(t=6.80, p_{\mathrm{FDR}}<.005 ; 1,091\right.$ voxels; Talairach coordinates: $10,50,36)$ and reward outcome $(t=$ $4.89, p_{\text {FDR }}<.05 ; 2,025$ voxels; Talairach coordinates: -4 , $52,34)$. Activation in the $\mathrm{mPFC}$ for reward anticipation was primarily in medial BA 9, although the coordinates of the maximum voxel in the cluster were in $\mathrm{BA} 8$; the reward outcome cluster included BAs 10 and 32 (see Figure 1B).

\section{Change in Symptoms Over Time}

Since the primary interest of this study was on treatment response, mixed-model analyses were estimated with the intercept reflecting discharge symptoms. Table 1 and Figure 2 present observed clinician ratings and selfreported symptom scores over the course of treatment. At discharge, the participants had residual symptoms, as indicated by significant mean intercept values or discharge levels (Table 2). Significant variance was found in discharge scores for general severity, depressive symptoms, and anxiety symptoms. Improvement scores did not have significant variance in intercept or slope and were, therefore, not included in analyses with fMRI data. 
Table 1

Descriptive Statistics for Clinician-Rated Severity and Improvement and Adolescents' Self-Reported Symptoms During an 8-Week Treatment for Depression

\begin{tabular}{|c|c|c|c|c|c|c|c|c|c|c|}
\hline \multirow[b]{2}{*}{ Measure } & \multicolumn{2}{|c|}{$\mathrm{T} 1(n=13)$} & \multicolumn{2}{|c|}{$\mathrm{T} 2(n=12)$} & \multicolumn{2}{|c|}{$\mathrm{T} 3(n=10)$} & \multicolumn{2}{|c|}{$\mathrm{T} 4(n=8)$} & \multicolumn{2}{|c|}{$\mathrm{T} 5(n=6)$} \\
\hline & $M$ & $S D$ & $M$ & $S D$ & $M$ & $S D$ & $M$ & $S D$ & $M$ & $S D$ \\
\hline Severity & 4.00 & 0.63 & 3.91 & 0.54 & 3.10 & 1.10 & 3.12 & 0.83 & 2.50 & 0.55 \\
\hline Improvement & & & 3.42 & 0.51 & 2.70 & 0.95 & 2.62 & 0.74 & 1.83 & 0.75 \\
\hline Depressive symptoms & 26.08 & 9.94 & 20.50 & 12.06 & 21.38 & 15.58 & 17.29 & 11.10 & 13.60 & 10.77 \\
\hline Anxiety symptoms & 29.83 & 19.48 & 27.40 & 17.21 & 26.12 & 14.99 & 23.29 & 12.23 & 18.40 & 11.26 \\
\hline
\end{tabular}

Note-T1, pretreatment; T2, Treatment Session 2; T3, Treatment Session 4; T4, Treatment Session 6; T5, Treatment Session 8 (posttreatment). Severity and improvement were assessed using the Clinical Global Impression-Severity and Clinical Global Impression-Improvement scales (Guy, 1976). Depressive and anxiety symptoms were assessed using the Mood and Feelings Questionnaire (Angold, Erkanli, Silberg, Eaves, \& Costello, 2002) and the Screen for Childhood Anxiety and Related Disorders (Birmaher et al., 1999), respectively. Improvement was not rated at T1 because it was the first session of treatment.

Table 2

Results of Growth Curve Model for Clinician-Rated Severity and Improvement and Adolescents' Self-Reported Symptoms During an 8-Week Treatment for Depression

\begin{tabular}{|c|c|c|c|c|c|c|c|c|}
\hline & \multicolumn{4}{|c|}{ Discharge Level } & \multicolumn{4}{|c|}{ Rate of Change } \\
\hline & $M$ & $S D$ & Var. & $S D$ & $M$ & $S E$ & Var. & $S E$ \\
\hline Severity & 2.74 & $0.26^{* * *}$ & 0.13 & $0.06^{*}$ & -0.13 & $0.04^{* *}$ & 0.00 & 0.00 \\
\hline Improvement & 1.99 & $0.39^{* * *}$ & 0.04 & 0.58 & -0.19 & $0.07^{* *}$ & 0.00 & 0.76 \\
\hline Depressive symptoms & 11.47 & $2.09^{* * *}$ & 88.69 & $2.32^{* * *}$ & -1.41 & $0.75^{+}$ & 0.00 & 59.48 \\
\hline Anxiety symptoms & 16.21 & $3.51^{* * *}$ & 97.29 & $35.15^{* *}$ & -1.53 & $0.49^{* *}$ & 2.41 & $1.38^{+}$ \\
\hline
\end{tabular}

Note- "Discharge Level" data are the mean and standard deviation of the model intercept, which reflects posttreatment level of each variable. "Rate of Change" data are the slope and standard error of the slope for each variable across treatment time points. Severity and improvement were assessed using the Clinical Global Impression-Severity and Clinical Global Impression-Improvement scales (Guy, 1976). Depressive and anxiety symptoms were assessed using the Mood and Feelings Questionnaire (Angold, Erkanli, Silberg, Eaves, \& Costello, 2002) and the Screen for Childhood Anxiety and Related Disorders (Birmaher et al., 1999), respectively. $\quad{ }^{+} p<.10 .{ }^{*} p<.05 .{ }^{* *} p<.01 . \quad{ }^{* * *} p<.001$.

The average rate of symptom reduction was significant for all measures, although the rate of change for depressive symptom scores was significant only at the level of a trend (Table 2). Rate of symptom change was similar for improvement, severity, and depressive symptoms. We found significant variance in reduction of anxiety symptoms. Figure 2 displays symptom scores observed over the course of treatment on the basis of these data.

\section{fMRI Predictors of Final Symptom Level and Change Over Time}

Next, associations between growth curve model parameters with significant variance in final symptom level (i.e., intercept) and rate of symptom reduction (i.e., slope) were examined. We focused on four types of reward-related brain function as predictors: striatal reactivity during reward anticipation, $\mathrm{mPFC}$ reactivity during reward anticipation, striatal reactivity during reward outcome, and $\mathrm{mPFC}$ reactivity during reward outcome.

For analyses with final symptom level, final general clinical severity was associated with striatal reactivity during reward outcome $(B=0.26, S E=0.08, p<.01)$. The final level of depressive symptoms was not associated with brain activation in either region during either task condition. The final level of anxiety symptoms was inversely associated with striatal reactivity during reward anticipation $(B=-4.70, S E=2.29, p<.05)$.
For analyses with change in symptoms over time, the rate of anxiety symptom reduction was inversely associated with striatal reactivity during reward anticipation $(B=$ $-0.64, S E=0.24, p<.01)$. This suggests that greater motivation-related striatal reactivity at pretreatment is associated with a faster rate of anxiety symptom decline over the course of treatment. In contrast, the rate of anxiety symptom reduction was positively associated with $\mathrm{mPFC}$ reactivity during reward anticipation $(B=4.21, S E=$ $1.50, p<.01)$. This suggests that greater motivationrelated $\mathrm{mPFC}$ reactivity at pretreatment is associated with a slower rate of anxiety symptom decline. Since there was not significant variance in the slope for depressive symptoms, overall improvement, or clinical severity, predictors of these slopes were not examined. ${ }^{2}$

\section{DISCUSSION}

The present study is the first to examine reward-related brain function as a predictor of treatment response in adolescent depression. In a sample of young people with MDD and high degree of anxiety disorder comorbidity, growth curve models indicated that the participants improved over time: During treatment, depressive symptoms, anxiety symptoms, and severity decreased (albeit at a trend level for depressive symptoms), and clinician-rated improvement increased. Greater reward-related striatal func- 
tion before treatment was associated with higher overall clinical severity at the end of treatment, lower anxiety symptoms at the end of treatment, and faster improvement in anxiety symptoms. Greater reward-related medial PFC function before treatment was related to slower improvement in anxiety symptoms. Reward-related brain function predicted final overall severity level, final anxiety symptom level, and rate of improvement of anxiety symptoms. This study is also unique in using fMRI in combination with growth curve modeling to account for patterns of change during treatment-including both final severity levels and slope of outcome measures over time-and their relation to brain function.

Heterogeneity in reward-related brain function among young people with depression may provide information on which people or which symptoms are likely to improve with treatment. Given that heterogeneity in response to treatment is considered the norm in depression (Brent et al., 2008), understanding the physiological markers that characterize potential responders could inform the development of new treatments or the personalization of existing treatments.

Findings that reward-related brain function predicted lower final anxiety symptoms and faster-decreasing anxiety symptoms are consistent with the hypothesis that higher - and potentially, more adaptive - reward-related brain function before treatment is associated with better treatment response. A possible explanation for this is that flexible reward responding, with its implications for affiliative behavior (Bora, Yücel, \& Allen, 2009), could serve as a mechanism in effective engagement in CBT. Such engagement could be expressed in the social, cognitive, or behavioral aspects of CBT. An adolescent with depression and comorbid anxiety who also responds to rewards might respond more collaboratively to the therapist, have optimism that the treatment will be helpful, be more motivated to try techniques suggested in treatment, experience more positive affect to behavioral activation, and have more awareness and enjoyment of positive changes in mood and experiences that occur with treatment. Along with an earlier study's findings on the positive clinical prognostic value of subjective positive affect in adolescents with depression (Joiner, Lewinsohn, \& Seeley, 2002), the present findings support the hypothesis that relatively higher positive affect could be protective in youth with depression and anxiety.

The present findings about the association between reward-related brain function and anxiety symptoms parallel, in some ways, other studies' findings that affectrelated brain function predicts treatment response. Most of the previous studies have focused on neural response to negative affect (Chen et al., 2007) or on threat-related brain systems (McClure et al., 2007), and although the present findings focus on reward-related, rather than threat-related, brain systems, the regulation of both systems is postulated to be altered in depression (Clark \& Watson, 1991; Fowles, 1988). Interestingly, anxiety is postulated to differ from depression in its weaker association with alterations in positive affect (Clark \& Watson,
1991), a construct that includes reward responding. That conceptual view is not supported by the present findings or by other findings on reward processing in anxiety traits or disorders (e.g., Forbes et al., 2006; Guyer et al., 2006). Perhaps appropriate affective responding, rather than blunted affective response, regardless of stimulus valence, promotes the ability to benefit from treatment. This hypothesis must be tested by examining both reward-related and threat-related brain function in a single sample and, ideally, in a single task. Our task did not allow this, so this question must be addressed by future studies.

Notably, depressive symptoms were not associated with reward-related brain function. The MFQ, our measure of depressive symptoms, is valuable as a depression screening tool and is more effective at detecting depressive than anxiety disorders (Katon et al., 2008). It is thus unlikely that the instrument measured anxiety symptoms better than it measured depressive symptoms. In growth curve models, low variability in slope for depressive symptoms over time limited our ability to test the relation between reward-related brain function and rate of change in depressive symptoms, and thus, we could not report associations between brain function and slope for depressive symptoms. This low variability could reflect a fairly uniform pattern of improvement in depressive symptoms among the adolescents in the present sample, and this similarity in pattern of improvement leaves little room for identifying factors related to improvement. In the present sample, anxiety symptoms had a more variable slope across time than did depressive symptoms, which could have been related to the presence of comorbid anxiety disorders in some participants. In addition, the difference between depressive symptom change and anxiety symptom change perhaps reflects the presence of more potential sources of influence on the improvement of anxiety with treatment.

The literature on anxiety disorders provides little context for our findings with anxiety symptoms. Because no previous studies have examined reward-related brain function as a predictor of treatment response in anxiety and few theoretical models of anxiety postulate alterations in reward functioning, it is difficult to interpret the association of reward-related brain function with change in anxiety symptoms. A possible explanation is that rewardrelated brain function is related to general affective psychopathology, rather than to depression in particular. Alternatively, the functioning of putative reward-related brain areas such as the striatum has yet to be determined definitively, and the striatum might be critical in some ways to the pathophysiology of anxiety. With the present pattern of findings, we cannot rule out the possibility that reward-related brain function is related more to treatment change involving anxiety than to that involving depression. Greater striatal reactivity has been reported in studies of adolescents with anxiety-related temperament (Guyer et al., 2006) or disorders (Forbes et al., 2006), and the next step in this line of research will be to directly compare the relation of reward-related brain function to treatment response in adolescents with anxiety only, depression only, and comorbid anxiety and depression. 
The relation of reward-related medial PFC reactivity to slower rate of improvement in anxiety symptoms is consistent, to some extent, with findings on medial PFC involvement in depression. In adults with depression, medial PFC reactivity to affective stimuli is enhanced (Sheline et al., 2009), and decreased resting medial PFC activity is associated with improvement after CBT (Goldapple et al., 2004). Also, adolescents with depression exhibit greater reward-related medial PFC reactivity (Forbes, Hariri, et al., 2009). Because the medial PFC is part of the default mode network, the association of mPFC rewardrelated reactivity with less rapid improvement in anxiety suggests that difficulty disengaging from the usual selffocused mental state interferes with response to treatment in adolescents with depression. The present association of medial PFC reactivity and change in anxiety symptoms could indicate that anxiety (at least in the context of comorbidity with depression) confers similar functional alterations to regions in the default mode network.

In contrast, the finding that greater overall clinical severity at the end of treatment was associated with higher striatal reactivity to reward was unexpected and does not support our hypothesis that greater reward-related responding predicts better treatment outcome. This correlation was not mirrored in the association between brain function and adolescents' symptoms: Reward-related brain function was unrelated to higher depressive symptoms at the end of treatment, and it was inversely related to anxiety symptoms. A difference between these measures is that overall clinical severity was rated by treatment providers, whereas symptoms were rated by the adolescents themselves. Treatment providers and adolescents could have different perspectives on the adolescents' symptoms and functioning, and differences in the instruments used for these variables are likely to lead them to capture different aspects of affective problems. It is also possible, of course, that higher reward-related brain function is related to the expression of distress to others, even if that distress is not experienced as meaningful by a young person. Furthermore, final severity was associated with striatal reactivity during the outcome condition. Adolescents who exhibit greater response to winning money might respond strongly to external stimuli in general, and as a result, they might be judged as having greater general clinical severity.

Our pattern of findings suggests that it is important to assess brain function in response to both reward anticipation and reward outcome when examining its predictive utility for treatment response. Greater anticipationrelated brain function was associated with lower anxiety symptom level at the end of treatment and faster change in anxiety symptoms during treatment. Improvement in anxiety might thus be particularly associated with reward anticipation, which is thought to represent motivation or wanting, rather than liking of rewards (Berridge \& Robinson, 2003).

Improvement in self-reported symptoms was generally similar to that for clinician-rated severity and improvement, but greater reward-related striatal reactivity predicted higher clinician-rated severity and lower selfreported anxiety. However, severity was related to BOLD response during reward outcome, and anxiety was related to response during reward anticipation. It is possible that these two aspects of reward processing - reward anticipation reflecting motivation and reward outcome reflecting enjoyment - are related to severity and anxiety in opposite ways.

Our findings must be considered in light of some limitations. Several of these were related to our small sample size. First, the sample size may have resulted in Type I errors in finding associations between brain activation and symptom scores and change (Maas \& Hox, 2005). In addition, not all the participants completed treatment, and missing data created a challenge for analyzing data for all time points for all participants. This was a result of dropout, and although we made extensive efforts to contact the participants and encourage completion of treatment, it may be valuable for future studies to use more creative methods to retain participants. Second, we were not able to compare psychosocial and combined psychosocial and pharmacotherapy treatments comprehensively. We could not include medication status in growth curve models, for example. Previous studies of adult depression have revealed that $\mathrm{CBT}$ and pharmacotherapy are associated with different patterns of treatment-related changes in brain function (Goldapple et al., 2004). It will be important for research on predictors and mechanisms of treatment response in young people to address possible differences in treatment modalities. Third, we had restricted ability to compare adolescents with and without comorbid anxiety disorders. We have found that depressed adolescents with anxiety disorders are similar in their reward-related brain function to those without comorbid anxiety disorders (Forbes, Hariri, et al., 2009), but we have also found, in a different sample, that anxiety symptoms are related to reward-related brain function independently of depressive symptoms (Forbes et al., 2006). It is important to examine comorbidity effects in the prediction of treatment response and to consider the possibility that comorbidity of anxiety and depression represents greater severity than does the presence of depression alone.

We acknowledge that our sample size was on the small side for growth curve modeling, but we also note that the number of time points in our study made growth curve modeling the most appropriate approach to examining change in symptoms, severity, and improvement over time. To address possible power issues for this technique, and guided by the small literature on sample size in multilevel modeling (Maas \& Hox, 2005), we conducted a Monte Carlo simulation in Mplus to examine the power to find significant variance estimates for the mean and variance components for the intercept and slope. We included the exact study characteristics from the present data to simulate 100 data sets to provide power estimates for the relevant parameters. Across outcome variables, power estimates for the intercept values, standard error of the intercept values, slope values, and standard error of the slope values were $.92, .37, .84$, and .34 , respectively. Thus, the 
present study was sufficiently powered to show significant intercept and slope values but had little power to identify significant variance in the intercept and slope parameters. Thus, it is impressive that we found significant variance in four of the eight growth parameters.

Several aspects of the study design also limit the scope of our findings. We collected fMRI data only before treatment, so we were not able to examine change in brain function with treatment. This issue is important in understanding the possible roles of reward-related brain function in both the pathophysiology of depression and the mechanisms of treatment response. One study has reported that reward-related areas have impaired functioning both before and after treatment in adult depression (Fu et al., 2007), which suggests that altered reward processing, at least in adults, is traitlike. Our study did not include a wait-list control group, which prevents us from concluding that change in severity reflects response to treatment rather than spontaneous recovery. Also, the high ratio of therapists to participants left us unable to examine potential therapist effects that could have contributed to variability in treatment response. Finally, treating clinicians, rather than independent evaluators, provided assessments of improvement and severity. Thus, some biases may have been present in their responses, even though ratings were performed without knowledge of the fMRI results.

Research on the pathophysiology and treatment of depression has indicated that development plays an important role in the disorder's onset and course. Findings in adolescents have not provided an exact replication of findings in adults, and developmental psychopathologists have postulated that the biological features of the disorder are different early in life (Kaufman, Martin, King, $\&$ Charney, 2001). Even though we did not find that age contributed to treatment response, the broad age range of the participants could have introduced other developmental effects into our study. We have found, in a different sample, that striatal response to reward decreases with pubertal maturation in healthy adolescents (Forbes et al., in press), and development of reward-related systems during adolescence could influence the onset, course, and treatment of depression. Therefore, future treatment studies of adolescents should employ designs that allow the careful examination of developmental factors in both the response to treatment over time and the prediction of treatment response from affect-related brain function.

In summary, this study yielded preliminary findings on reward-related brain function as a predictor of treatment response in adolescents with depression, many of whom had comorbid anxiety disorders. The findings point to the potential importance of reward-related brain function in the treatment of depression, not simply in its pathophysiology. With a more complete understanding of the types of affect-related brain function that indicate which young people with depression will likely have a favorable response to treatment, it will become more possible to improve the efficacy of existing treatments or to develop biologically guided treatments that are personalized.

\section{AUTHOR NOTE}

This study was supported by an NIMH Program Project (P01 MH41712; N.D.R., principal investigator [PI]), an NIMH Mentored Research Scientist Development Award (K01 MH074769, E.E.F., PI), an NIMH Training Grant (T32 MH018951; David A. Brent, PI), and a NARSAD Young Investigator Award (E.E.F., PI). We are grateful to Alex Johnston and Kelsey Ronan for assistance with data analyses and figures. We thank the staff of the Child and Adolescent Sleep and Neurobehavioral Laboratory, the staff of the STAR clinic, and the families who participated in the study. Correspondence concerning this article should be addressed to E. E. Forbes, Western Psychiatric Institute and Clinic, Loeffler 319, 3811 O'Hara Street, Pittsburgh, PA 15213 (e-mail: forbese@upmc.edu).

\section{REFERENCES}

Angold, A. (1988). Childhood and adolescent depression: I. Epidemiological and aetiological aspects. British Journal of Psychiatry, 152, 601-617.

Angold, A., Erkanli, A., Silberg, J., Eaves, L., \& Costello, E. J. (2002). Depression scale scores in 8-17-year-olds: Effects of age and gender. Journal of Child Psychology \& Psychiatry, 43, 10521063.

Bar-Haim, Y., Fox, N. A., Benson, B., Guyer, A. E., Williams, A., Nelson, E. E., ET AL. (2009). Neural correlates of reward processing in adolescents with a history of inhibited temperament. Psychological Science, 20, 1009-1018.

Berridge, K. C., \& Robinson, T. E. (2003). Parsing reward. Trends in Neurosciences, 26, 507-513.

Birmaher, B., Brent, D. A., Chiappetta, L., Bridge, J., Monga, S., \& Baugher, M. (1999). Psychometric properties of the Screen for Child Anxiety Related Emotional Disorders (SCARED): A replication study. Journal of the American Academy of Child \& Adolescent Psychiatry, 38, 1230-1236.

Bora, E., YüCEl, M., \& Allen, N. B. (2009). Neurobiology of human affiliative behaviour: Implications for psychiatric disorders. Current Opinion in Psychiatry, 22, 320-325.

Brent, D. [A.], Emslie, G., Clarke, G., Wagner, K. D., Asarnow, J. R., Keller, ET AL. (2008). Switching to another SSRI or to venlafaxine with or without cognitive behavioral therapy for adolescents with SSRI-resistant depression: The TORDIA randomized controlled trial. JAMA, 299, 901-913.

Brent, D. A., Holder, D., Kolko, D., Birmaher, B., Baugher, M., Roth, C., ET AL. (1997). A clinical psychotherapy trial for adolescent depression comparing cognitive, family, and supportive therapy. $A r-$ chives of General Psychiatry, 54, 877-885.

Chen, C.-H., Ridler, K., Suckling, J., Williams, S., Fu, C. H. Y., Merlo-Pich, E., \& Bullmore, E. (2007). Brain imaging correlates of depressive symptom severity and predictors of symptom improvement after antidepressant treatment. Biological Psychiatry, 62, 407-414.

Clark, L. A., \& Watson, D. (1991). Tripartite model of anxiety and depression: Psychometric evidence and taxonomic implications. Journal of Abnormal Psychology, 100, 316-336.

Costafreda, S. G., Khanna, A., Mourao-Miranda, J., \& Fu, C. H. Y. (2009). Neural correlates of sad faces predict clinical remission to cognitive behavioural therapy in depression. NeuroReport, 20, 637 641.

Davey, C. G., Yücel, M., \& Allen, N. B. (2008). The emergence of depression in adolescence: Development of the prefrontal cortex and the representation of reward. Neuroscience \& Biobehavioral Reviews, 32, 1-19.

Delgado, M. R., Nystrom, L. E., Fissell, C., Noll, D. C., \& Fiez, J. A. (2000). Tracking the hemodynamic responses to reward and punishment in the striatum. Journal of Neurophysiology, 84, 30723077.

Dunlop, B. W., \& NemerofF, C. B. (2007). The role of dopamine in the pathophysiology of depression. Archives of General Psychiatry, 64, 327-337.

Epstein, J., Pan, H., Kocsis, J. H., Yang, Y., Butler, T., Chusid, J., ET AL. (2006). Lack of ventral striatal response to positive stimuli in 
depressed versus normal subjects. American Journal of Psychiatry, 163, 1784-1790.

Forbes, E. E., Brown, S. M., Kimak, M., Ferrell, R. E., Manuck, S. B., \& HARIRI, A. R. (2009). Genetic variation in components of dopamine neurotransmission impacts ventral striatal reactivity associated with impulsivity. Molecular Psychiatry, 14, 60-70.

Forbes, E. E., \& DAHL, R. E. (2005). Neural systems of positive affect: Relevance to understanding child and adolescent depression? Development \& Psychopathology, 17, 827-850.

Forbes, E. E., Hariri, A. R., Martin, S. L., Silk, J. S., Moyles, D. L., Fisher, P. M., ET AL. (2009). Altered striatal activation predicting real-world positive affect in adolescent major depressive disorder. American Journal of Psychiatry, 166, 64-73.

Forbes, E. E., May, C. J., Siegle, G. J., Ladouceur, C. D., Ryan, N. D., CARTER, C. S., ET AL. (2006). Reward-related decision-making in pediatric major depressive disorder: An fMRI study. Journal of Child Psychology \& Psychiatry, 47, 1031-1040.

Forbes, E. E., Ryan, N. D., Phillips, M. L., Manuck, S. B., WorthMAN, C. M., MoyLes, D. L., ET AL. (in press). Reward-related brain function in adolescents: Role of puberty and relation to positive affect and depressive symptoms. Journal of the American Academy of Child and Adolescent Psychiatry.

Fowles, D. C. (1988). Psychophysiology and psychopathology: A motivational approach. Psychophysiology, 25, 373-391.

Fu, C. H., Williams, S. C., Brammer, M. J., Suckling, J., Kim, J., Cleare, A. J., ET AL. (2007). Neural responses to happy facial expressions in major depression following antidepressant treatment. American Journal of Psychiatry, 164, 599-607.

Fu, C. H., Williams, S. C., Cleare, A. J., Scott, J., MitterschiffthaLER, M. T., WALSh, N. D., ET AL. (2008). Neural responses to sad facial expressions in major depression following cognitive behavioral therapy. Biological Psychiatry, 64, 505-512.

Goldapple, K., Segal, Z., Garson, C., Lau, M., Bieling, P., KenNedY, S., \& MaYberg, H. (2004). Modulation of cortical-limbic pathways in major depression: Treatment-specific effects of cognitive behavior therapy. Archives of General Psychiatry, 61, 34-41.

Gross, J. J., \& MuÑoz, R. (1995). Emotion regulation and mental health. Clinical Psychology: Science \& Practice, 2, 151-164.

GuY, W. (1976). Clinical global impressions. In W. Guy (Ed.), ECDEU assessment manual for psychopharmacology, revised (pp. 218-222). Rockville, MD: National Institute of Mental Health.

Guyer, A. E., Nelson, E. E., Perez-Edgar, K., Hardin, M. G., Roberson-NaY, R., Monk, C. S., ET AL. (2006). Striatal functional alteration in adolescents characterized by early childhood behavioral inhibition. Journal of Neuroscience, 26, 6399-6405.

JoIner, T. E., JR., LeWinsohn, P. M., \& SeELey, J. R. (2002). The core of loneliness: Lack of pleasurable engagement-more so than painful disconnection - predicts social impairment, depression onset, and recovery from depressive disorders among adolescents. Journal of Personality Assessment, 79, 472-491.

Katon, W., Russo, J., Richardson, L., McCauley, E., \& Lozano, P. (2008). Anxiety and depression screening for youth in a primary care population. Ambulatory Pediatrics, 8, 182-188.

Kaufman, J., Birmaher, B., Brent, D., Rao, U., Flynn, C., Moreci, P., ET AL. (1997). Schedule for affective disorders and schizophrenia for school-age children-Present and lifetime version (K-SADS-PL): Initial reliability and validity data. Journal of the American Academy of Child \& Adolescent Psychiatry, 36, 980-988.

Kaufman, J., Martin, A., King, R. A., \& Charney, D. (2001). Are child-, adolescent-, and adult-onset depression one and the same disorder? Biological Psychiatry, 49, 980-1001.

Keedwell, P. A., Andrew, C., Williams, S. C., Brammer, M. J., \& Phillips, M. L. (2005). A double dissociation of ventromedial prefrontal cortical responses to sad and happy stimuli in depressed and healthy individuals. Biological Psychiatry, 58, 495-503.

Коов, G. F., \& Le MoAL, M. (2008). Neurobiological mechanisms for opponent motivational processes in addiction. Philosophical Transactions of the Royal Society B, 363, 3113-3123.

Kring, A. M., Persons, J. B., \& Thomas, C. (2007). Changes in affect during treatment for depression and anxiety. Behaviour Research \& Therapy, 45, 1753-1764.

Lewinsohn, P. M., Clarke, G. N., Seeley, J. R., \& Rohde, P. (1994).
Major depression in community adolescents: Age at onset, episode duration, and time to recurrence. Journal of the American Academy of Child \& Adolescent Psychiatry, 33, 809-818.

Lewinsohn, P. M., Rohde, P., Klein, D. N., \& Seeley, J. R. (1999). Natural course of adolescent major depressive disorder: I. Continuity into young adulthood. Journal of the American Academy of Child \& Adolescent Psychiatry, 38, 56-63.

Lutz, W., Martinovich, Z., \& Howard, K. I. (1999). Patient profiling: An application of random coefficient regression models to depicting the response of a patient to outpatient psychotherapy. Journal of Consulting \& Clinical Psychology, 67, 571-577.

MaAs, C. J. M., \& Hox, J. J. (2005). Sufficient sample sizes for multilevel modeling. Methodology, 1, 86-92.

March, J., Silva, S., Petrycki, S., Curry, J., Wells, K., Fairbank, J., ET AL. (2004). Fluoxetine, cognitive-behavioral therapy, and their combination for adolescents with depression: Treatment for Adolescents With Depression Study (TADS) randomized controlled trial. JAMA, 292, 807-820.

McClure, E. B., Adler, A., Monk, C. S., Cameron, J., Smith, S., Nelson, E. E., ET AL. (2007). fMRI predictors of treatment outcome in pediatric anxiety disorders. Psychopharmacology, 191, 97-105.

Muthén, L. K., \& Muthén, B. O. (2006). Mplus user's guide (4th ed.). Los Angeles: Muthén \& Muthén.

Olino, T. M., Klein, D. N., Lewinsohn, P. M., Rohde, P., \& SeeLEY, J. R. (2008). Longitudinal associations between depressive and anxiety disorders: A comparison of two trait models. Psychological Medicine, 38, 353-363.

RAICHLE, M. E., \& SNYDER, A. Z. (2007). A default mode of brain function: A brief history of an evolving idea. NeuroImage, 37, 1083-1090; discussion, 1097-1089.

Raudenbush, S. W., \& BRYK, A. S. (2002). Hierarchical linear models: Applications and data analysis methods. Thousand Oaks, CA: Sage.

Salvadore, G., Cornwell, B. R., Colon-Rosario, V., Coppola, R., Grillon, C., Zarate, C. A., JR., \& ManjI, H. K. (2009). Increased anterior cingulate cortical activity in response to fearful faces: A neurophysiological biomarker that predicts rapid antidepressant response to ketamine. Biological Psychiatry, 65, 289-295.

Schlaepfer, T. E., Cohen, M. X., Frick, C., Kosel, M., Brodesser, D., AXMACHER, N., ET AL. (2008). Deep brain stimulation to reward circuitry alleviates anhedonia in refractory major depression. Neuropsychopharmacology, 33, 368-377.

Sheline, Y. I., Barch, D. M., Donnelly, J. M., Ollinger, J. M., SnYDER, A. Z., \& MinTUn, M. A. (2001). Increased amygdala response to masked emotional faces in depressed subjects resolves with antidepressant treatment: An fMRI study. Biological Psychiatry, 50, 651-658.

Sheline, Y. I., Barch, D. M., Price, J. L., Rundle, M. M., Vaishnavi, S. N., SNyder, A. Z., ET AL. (2009). The default mode network and self-referential processes in depression. Proceedings of the National Academy of Sciences, 106, 1942-1947.

Siegle, G. J., Carter, C. S., \& Thase, M. E. (2006). Use of FMRI to predict recovery from unipolar depression with cognitive behavior therapy. American Journal of Psychiatry, 163, 735-738.

Silk, J. S., Forbes, E. E., Whalen, D. J., JAKubCaK, J. L., Ryan, N. D., AXELSON, D., ET AL. (2010). Ecological momentary assessment of child and adolescent depression: Daily emotional and social dynamics. Manuscript under review.

Spielmans, G. I., \& McFall, J. P. (2006). A comparative meta-analysis of clinical global impressions change in antidepressant trials. Journal of Nervous \& Mental Disease, 194, 845-852.

Surguladze, S., Brammer, M. J., Keedwell, P., Giampietro, V., Young, A. W., Travis, M. J., ET AL. (2005). A differential pattern of neural response toward sad versus happy facial expressions in major depressive disorder. Biological Psychiatry, 57, 201-209.

Tremblay, L. K., Naranjo, C. A., Graham, S. J., Herrmann, N., Mayberg, H. S., Hevenor, S., \& Busto, U. E. (2005). Functional neuroanatomical substrates of altered reward processing in major depressive disorder revealed by a dopaminergic probe. Archives of General Psychiatry, 62, 1228-1236.

Weissman, M. M., Wolk, S., Goldstein, R. B., Moreau, D., Adams, P., Greenwald, S., ET AL. (1999). Depressed adolescents grown up. $J A M A, \mathbf{2 8 1}, 1707-1713$. 
Whalen, P. J., Johnstone, T., Somerville, L. H., Nitschke, J. B., Polis, S., Alexander, A. L., ET AL. (2008). A functional magnetic resonance imaging predictor of treatment response to venlafaxine in generalized anxiety disorder. Biological Psychiatry, 63, 858-863.

Willett, J. B. (1997). Measuring change: What individual growth modeling buys you. In E. Amsel \& K. A. Renninger (Eds.), Change and development: Issues of theory, method, and application (pp. 213243). Mahwah, NJ: Erlbaum.

Williamson, D. E., Forbes, E. E., Dahl, R. E., \& Ryan, N. D. (2005). A genetic epidemiologic perspective on comorbidity of depression and anxiety. Child \& Adolescent Psychiatric Clinics of North America, 14, 707-726.

Wood, A., Kroll, L., Moore, A., \& Harrington, R. (1995). Properties of the mood and feelings questionnaire in adolescent psychiatric outpatients: A research note. Journal of Child Psychology \& Psychiatry, 36, 327-334.

\section{NOTES}

1. To complement growth models of treatment response and its relation to reward-related brain function, pre-/posttreatment analyses were also conducted, using change scores computed by subtracting the pretreatment severity level from the posttreatment level. When no data were available from Week 9 of treatment, difference scores were computed on the basis of the most distant intervals available for each participant (e.g., Week 7 vs. Week 1). Analyses were conducted for clinician-rated severity, self-reported depressive symptoms, and self-reported anxiety symptoms. Analyses were not conducted for clinician-rated improvement, since this is not rated at baseline and represents a change score. As is indicated in the following correlation table, pre-/posttreatment analyses revealed trend-level associations between increased $\mathrm{mPFC}$ activation during anticipation of reward and less improvement in depressive and anxiety symptoms. The association with change in anxiety symptoms is similar to our growth model finding that $\mathrm{mPFC}$ activation during anticipation was associated with a slower rate of anxiety symptom reduction.

\begin{tabular}{|c|c|c|c|c|c|c|}
\hline & \multicolumn{6}{|c|}{ Change Scores (Pre- to Posttreatment) } \\
\hline & \multicolumn{2}{|c|}{ Severity } & \multicolumn{2}{|c|}{$\begin{array}{l}\text { Depressive } \\
\text { Symptoms }\end{array}$} & \multicolumn{2}{|c|}{$\begin{array}{c}\text { Anxiety } \\
\text { Symptoms }\end{array}$} \\
\hline & $r$ & $p$ & $r$ & $p$ & $r$ & $p$ \\
\hline Anticipation $\mathrm{mPFC}$ & -.29 & .36 & -.52 & .09 & -.51 & .09 \\
\hline Anticipation striatum & .14 & .66 & .13 & .69 & .29 & .36 \\
\hline Outcome mPFC & -.05 & .87 & -.21 & .51 & -.15 & .63 \\
\hline Outcome striatum & -.16 & .63 & -.25 & .44 & -.08 & .81 \\
\hline
\end{tabular}

Note-mPFC, medial prefrontal cortex.

2. Additional growth curve analyses of brain function as a predictor of treatment response were conducted with fMRI data from the entire reward task. Findings from these analyses supported the claim that reward-related brain function predicts treatment response in adolescent depression, but the pattern of findings differed from that of the growth curve models with data from the first run of the task. Specifically, general clinical severity at discharge was predicted by striatal reactivity during reward anticipation $(B=0.47, S E=0.16, p<.01)$, and anxiety symptoms at discharge were predicted by $\mathrm{mPFC}$ reactivity during both anticipation $(B=-68.49, S E=21.25, p<.01)$ and outcome $(B=$ $-37.84, S E=9.56, p<.001)$. Depressive symptoms at discharge and rate of change of anxiety symptoms were not associated with striatal or $\mathrm{mPFC}$ reactivity during reward anticipation or reward outcome.

(Manuscript received May 11, 2009; revision accepted for publication August 16, 2009.) 